\title{
Lack of Effect of the 5-HT 4 Receptor Ligands RS 67333 and RS 39604 on Murine Agonistic Behaviour
}

\author{
Robert Bell, Karl Lynch \\ School of Psychology, The Queen's University of Belfast, Belfast, Northern Ireland \\ Email: r.bell@qub.ac.uk
}

Received August 31, 2011; revised October 13, 2011; accepted November 11, 2011

\begin{abstract}
In comparison to studies investigating the roles of $5-\mathrm{HT}_{1}, 5-\mathrm{HT}_{2}$ and $5-\mathrm{HT}_{3}$ receptors in aggressive behaviour there is a dearth of material examining the function of $5-\mathrm{HT}_{4}$ receptors in this behaviour. In view of this, the current study examined the effects of the 5- $\mathrm{HT}_{4}$ receptor partial agonist RS 67333 and antagonist RS 39604 in murine agonistic behaviour. RS 67333 failed to produce any significant changes in the offensive. Significant variation in the frequency of evade behaviour was detected but this occurred between treatment groups rather than with controls. Interestingly, both the frequency and duration of stretched attend behaviour were increased by RS $673330.1 \mathrm{mg} / \mathrm{kg}$, a result indicative of increased risk assessment. The administration of RS $39604(0.01-1 \mathrm{mg} / \mathrm{kg})$ produced significant variation in the frequency and duration of following, and aggressive grooming. Frozen crouch behaviour was also increased significantly at $0.1 \mathrm{mg} / \mathrm{kg}$. It is concluded that since the $5-\mathrm{HT}_{4}$ receptor ligands employed in this study produced very few significant behavioural effects across the treatment groups, $5-\mathrm{HT}_{4}$ receptors do not play a role in the modulation of murine aggressive behaviour.
\end{abstract}

Keywords: RS 67333; RS 39604; Agonistic Behaviour; Social Behaviour; Ethological Analysis

\section{Introduction}

Compared to the early extensive research interest that focused on the role of 5- $\mathrm{HT}_{1}$ receptors [1-3] and 5- $\mathrm{HT}_{2}$ receptors [4] in rodent offensive behaviour, to the best of the authors' knowledge no studies have been published investigating the role of the $5-\mathrm{HT}_{4}$ receptor in aggressive behaviour.

However, the 5- $\mathrm{HT}_{4}$ receptor partial agonist RS 67333 has been tested in other animal models of behaviour. In the Morris Water maze the partial agonist RS 67333 produced significant reductions in the cognitive deficit induced by atropine and while the partial agonist RS 67506 produced no significant change in this respect [5]. Testing also showed that RS 67333 produced no effect on locomotor activity while the antagonist RS 67532 (1 \& $10 \mathrm{mg} / \mathrm{kg}$ i.p.) did reduce activity. More recently, in the forced swim test animal model of depression, RS 67333 demonstrated putative antidepressant action [6]; an effect optimized by the adjunction of an SSRI [7].

$5-\mathrm{HT}_{4}$ receptors have been examined with regard to a possible role in anxiety. The $5-\mathrm{HT}_{4}$ antagonists SB204070 and GR113808 were tested in the elevated plus maze [8]. The results were suggestive of a mild anxiolytic-like effect, although not as potent as traditional anxiolytics such as diazepam.
An investigation concerning the role of $5-\mathrm{HT}_{4}$ receptors in appetitive behaviour reported that bilateral microinjections of the 5- $\mathrm{HT}_{4}$ antagonist RS 39604 into the nucleus accumbens (NAc) increased food intake and increased weight loss in MDMA treated rats compared to saline controls. Such results demonstrated that $5-\mathrm{HT}_{4}$ receptors in the NAc regulate the apetite suppressant effects of MDMA [9].

In this study we examined the effects of the $5-\mathrm{HT}_{4}$ partial agonist RS 67333 and the 5-HT 4 antagonist RS 39604 on murine agonistic behaviour.

\section{Materials and Methods}

Subjects and procedure. One hundred and sixty adult male albino mice of the BKW strain, weighing between 25 - 35 g from Queens University Belfast Medical Biology Centre Breeding stock were used. Four weeks prior to testing the mice were randomly allocated to resident or intruder status. Residents were individually caged (cage size $30 \times 15 \times 13 \mathrm{~cm})$ and intruders housed with siblings in groups of approximately ten (cage size $44 \times 28 \times 13$ $\mathrm{cm})$. Throughout the four weeks prior to testing, all animals were given fresh bedding weekly, with food and water available ad libitum. All subjects were maintained in a temperature-controlled room $\left(24^{\circ} \mathrm{C} \pm 1^{\circ} \mathrm{C}\right)$, in which 
a 12 hour reversed light/dark cycle was operative (lights on $2400 \mathrm{hrs}$ ).

All work was licensed by the Home Office in accordance with the UK Animals (Scientific Procedures) Act 1986.

Behavioural testing took place in the residents "home cage". Food and water were removed from test cages for the duration of encounters. Resident/intruder encounters were recorded on tape by a Panasonic Saticon colour video camera (model WVP200E) with low light facility. The test cages were illuminated by two 60watt "anglepoise" lamps during social encounters. Tape analysis was carried out using a Panasonic video recorder, a VDU, a $\mathrm{PC}$ computer and a printer.

All injections were performed subcutaneously in a volume of $10 \mathrm{ml} / \mathrm{kg}$ thirty minutes prior to testing. Animals used were both drug and experimentally naive. The experimenter remained blind to the conditions until data analysis was complete.

All testing was carried out under red light during the mid-portion of the dark phase. Isolates were weighed, marked for recognition and randomly assigned to dose treatment groups. Only isolate (resident) mice received drug treatments. Thirty minutes after treatment (residents), intruder mice were introduced into the home cages of the residents and the ensuing 10 minute encounters recorded on video tape for later analysis.

RS 39604 was administered in doses of $0.01 \mathrm{mg} / \mathrm{kg}$, $0.1 \mathrm{mg} / \mathrm{kg}$ and $1 \mathrm{mg} / \mathrm{kg}$ and RS 67333 was administered in doses of $0.01 \mathrm{mg} / \mathrm{kg}, 0.1 \mathrm{mg} / \mathrm{kg} \& 0.5 \mathrm{mg} / \mathrm{kg}$. Doses were based on similar compounds from previous studies and pilot experiments in our laboratory.

\section{Measures}

Behavioural analysis was similar to that previously described [1]. Briefly, videotapes were analysed using direct keyboard inputs to the microcomputer which had been programmed to produce data output in the form of frequency and real-time duration of behavioural elements [3]. The following behavioural elements and categories were analysed:

\section{Statistical Analysis}

Given the non-parametric nature of the data, results for each behavioural element were analysed using KruskalWallis one-way analyses of variance. Where statistical differences were detected, further comparisons (with control group) were performed by Mann-Whitney U tests.

\section{Results}

\subsection{Resident Behaviours after Treatment with RS 67333 (0.01 - 0.5 mg/kg) (Table 1)}

\subsubsection{RS 67333 and non Social Behaviour}

Treatment with SR57227A sc. failed to significantly mo- dify non social behaviour on behalf of the drug treated resident mice with non significant increases and decreases in these behaviours being detected $(\mathrm{H} \leq 7.3, \mathrm{p}>0.05$ [f] \& $\mathrm{H} \leq 5.7, \mathrm{p}>0.05$ [d]).

\subsubsection{RS 67333 and Social Behaviour}

Kruskal Wallis analysis did detect significant variation in both the frequency and duration of stretched attend behaviour $(\mathrm{H}=12.6, \mathrm{p}<0.02$ [f] \& $\mathrm{H} \leq 9.9, \mathrm{p}>0.02$ [d] $)$. Subsequent analysis failed to showed a significant increase in both the frequency and duration of this behaviour at $0.1 \mathrm{mg} / \mathrm{kg}(\mathrm{p}<0.02)$. Analysis of the other elements of the social repertoire revealed no significant changes in behaviour $(\mathrm{H} \leq 6.2, \mathrm{p}>0.05$ [f] \& $\mathrm{H} \leq 4.8, \mathrm{p}>0.05$ [d]).

\subsubsection{RS 67333 and Offensive Behaviour}

The frequency and duration of offensive aggression and threat behaviour were not modified by treatment with RS 67333 with non significant changes being detected $(\mathrm{H} \leq$ $4.3, p>0.05[\mathrm{f}] \&[\mathrm{~d}])$.

\subsubsection{RS 67333 and Defensive Behaviour}

Kruskal Wallis analysis did detect significant variation in the frequency of evade behaviour $(\mathrm{H}=8.8, \mathrm{p}<0.05)$. Subsequent Mann Whitney analysis showed this variation to occur within the drug treated groups and not with the control. All other elements failed to show significant variation.

\subsection{Intruder Behaviours as a Function of Resident Behaviour after Treatment with RS 67333 (0.01 - 0.5 mg/kg) (Table 2)}

\subsubsection{RS 67333 and Intruder non Social Behaviour}

Kruskal Wallis analysis revealed no aspects of intruder non social behaviour were modified by RS $67333(\mathrm{H} \leq$ 2.6, $\mathrm{p}>0.05[\mathrm{f}] \& \mathrm{H} \leq 2.4, \mathrm{p}>0.05)$.

\subsubsection{RS 67333 and Intruder Social Behaviour}

Only one aspect of intruder social behaviour was significantly modified by the administration of RS 67333 . The frequency of naso naso sniffing showed significant modification $\mathrm{H}=8.7, \mathrm{p}<0.05)$ and was increased at 0.01 $\mathrm{mg} / \mathrm{kg}(\mathrm{p}<0.05)$.

No other elements of intruder social behaviour showed any significant change across the dose range with only small increases and decreases in behaviour being detected.

\subsubsection{RS 67333 and Intruder Offensive Behaviour}

Analysis of the intruder offensive behaviour revealed significant variation for the frequency and duration of offensive upright attacks $(\mathrm{H}+12.3, \mathrm{p}<0.02$ [f] \& $\mathrm{H}=10.8$, $\mathrm{p}<0.02$ [d] $)$ and chase behaviours $(\mathrm{H}=12.6, \mathrm{p}<0.02$ [f] $\& H=14, p<0.02[d])$. 
Table 1. Effects of RS $67333(0.01-0.5 \mathrm{mg} / \mathrm{kg})$ on behaviours displayed by resident mice.

\begin{tabular}{|c|c|c|c|c|c|c|}
\hline Behaviours & & Vehicle & $0.01 \mathrm{mg} / \mathrm{kg}$ & $0.1 \mathrm{mg} / \mathrm{kg}$ & $0.5 \mathrm{mg} / \mathrm{kg}$ & $H$ values \\
\hline \multirow[t]{2}{*}{ Cage exploration } & $\mathrm{f}$ & $41.5(32-50.75)$ & $25.5(13.5-36.5)$ & $37(28.5-53)$ & $34.5(25.75-40.25)$ & 7.3 \\
\hline & $\mathrm{d}$ & $181.38(137.82-203.97)$ & $143.14(60-216.42)$ & $152.17(105.56-253.67)$ & $227.24(157.97-266.91)$ & 4.4 \\
\hline \multirow[t]{2}{*}{ Rearing } & $\mathrm{f}$ & $30.5(11.5-36.25)$ & $15(3.75-29)$ & $16.5(7.75-22.75)$ & $19(15.25-36.75)$ & 4.5 \\
\hline & $\mathrm{d}$ & $68.65(25.03-82.7)$ & $26.34(5.15-73.36)$ & $26.43(10.57-35.65)$ & $56.83(20.99-121.13)$ & 5.7 \\
\hline \multirow{2}{*}{ Maintenance } & $\mathrm{f}$ & $2.5(.75-4.25)$ & $3(1-5)$ & $1(1-3)$ & $2(0.75-3.25)$ & 2.9 \\
\hline & $\mathrm{d}$ & $8.82(1.57-23.51)$ & $11.27(5.05-24.40)$ & $4.87(0.54-10.262)$ & $6.46(1.44-15.51)$ & 2.3 \\
\hline \multirow[t]{2}{*}{ Digging } & $\mathrm{f}$ & $0(0-0.25)$ & $0(0-1)$ & $0.5(0-1.25)$ & $0(0-2)$ & 2 \\
\hline & $\mathrm{d}$ & $0(0-0.1)$ & $0(0-1.13)$ & $0.5(0-1.74)$ & $0(0-5.37)$ & 2.2 \\
\hline \multirow[t]{2}{*}{ Naso genital } & $\mathrm{f}$ & $10.5(6.5-15)$ & $5(.75-11.25)$ & $7.5(3-18)$ & $6(2-8.25)$ & 4.7 \\
\hline & $\mathrm{d}$ & $36.33(14.82-72.23)$ & $16.89(0.5-46.99)$ & $26.33(10.7-67.21)$ & $14.47(4.55-41.86)$ & 2.7 \\
\hline \multirow[t]{2}{*}{ Naso nasal } & $\mathrm{f}$ & $11(8-17.5)$ & $14.5(10-18.75)$ & $15.5(10.5-18)$ & $10(6-11.25)$ & 6.2 \\
\hline & $\mathrm{d}$ & $38.68(26.94-75.40)$ & $57.99(30.79-72.01)$ & $49.60(31.43-63.92)$ & $27.1(18.1-51.16)$ & 3.5 \\
\hline Non specific & $\mathrm{f}$ & $13(9.75-19)$ & $6(3.75-10.75)$ & $11(4.75-17)$ & $10(6.25-15.75)$ & 3.6 \\
\hline investigation & $\mathrm{d}$ & $45.1(29.41-81.81)$ & $20.54(12.28-52)$ & $27(12.68-40.98)$ & $26.75(16.97-63.20)$ & 4.8 \\
\hline \multirow[t]{2}{*}{ Attend } & $\mathrm{f}$ & $9.5(5.75-12)$ & $5.5(1-11.25)$ & $10(2-12.75)$ & $6.5(3-10)$ & 1.4 \\
\hline & $\mathrm{d}$ & $15.36(8.9-16.64)$ & $11.71(1.84-15.66)$ & $14.4(6.33-19.24)$ & $10.79(5.08-17.27)$ & 2.3 \\
\hline \multirow[t]{2}{*}{ Stretched attend } & $\mathrm{f}$ & $4.5(2.75-7.25)$ & $10.5(6.25-13.5)$ & $13(7.5-22)$ & $3(1-8.25)$ & 12.6 \\
\hline & $\mathrm{d}$ & $5.41(3.05-13.22)$ & $14.26(6.02-25.06)$ & $21.53(10.30-35.65)$ & $5.55(1.19-12.74)$ & 9.9 \\
\hline \multirow[t]{2}{*}{ Follow } & $\mathrm{f}$ & $3.5(1.75-8.25)$ & $1(0-3)$ & $2(0.75-5.75)$ & $3(1-7.25)$ & 3.3 \\
\hline & d & $6.04(3.38-18.64)$ & $1.81(0-14.36)$ & $3.48(0.7-10.56)$ & $6.97(1.79-16.36)$ & 3.2 \\
\hline \multirow[t]{2}{*}{ Aggressive groom } & $\mathrm{f}$ & $0(0-0)$ & $0(0-0.5)$ & $0(0-0.25)$ & $0(0-0)$ & 4.3 \\
\hline & $\mathrm{d}$ & $0(0-0)$ & $0(0-3.5)$ & $0(0-0.51)$ & $0(0-0)$ & 4.3 \\
\hline \multirow[t]{2}{*}{ Tail rattle } & $\mathrm{f}$ & $8.5(1.75-15.5)$ & $11(.75-25.5)$ & $7.5(2.75-20.75)$ & $5.5(1.5-18.5)$ & 0.5 \\
\hline & $\mathrm{d}$ & $17.27(1.75-31.05)$ & $11.21(0.46-44.37)$ & $9.07(2.59-32.01)$ & $11.13(2.89-34.01)$ & 0.2 \\
\hline \multirow[t]{2}{*}{ Offensive sideways } & $\mathrm{f}$ & $8(1.75-29)$ & $14(0-34.5)$ & $19.5(1.75-25.5)$ & $9(2.5-18.75)$ & 0.4 \\
\hline & $\mathrm{d}$ & $35.63(1.01-105.54)$ & $24.92(0-78.40)$ & $48.69(6.06-79.19)$ & $26.73(8.79-56.63)$ & 0.8 \\
\hline \multirow[t]{2}{*}{ Offensive upright } & $\mathrm{f}$ & $2(0-4)$ & $3(0-5.5)$ & $2.5(0.75-6.75)$ & $3(0.75-5)$ & 0.9 \\
\hline & $\mathrm{d}$ & $4.92(0-17.73)$ & $4.39(0-7.3)$ & $4.78(0.66-14.52)$ & $5.68(1.52-9.22)$ & 0.5 \\
\hline \multirow[t]{2}{*}{ Chase } & $\mathrm{f}$ & $0(0-1.25)$ & $0(0-1)$ & $0.5(0-1.25)$ & $0(0-1.25)$ & 1.1 \\
\hline & d & $0(0-0.87)$ & $0(0-0.46)$ & $0.16(0-1.57)$ & $0(0-1.46)$ & 0.8 \\
\hline \multirow[t]{2}{*}{ Bite attack } & $\mathrm{f}$ & $6(0-20.25)$ & $16.5(0-39.25)$ & $9.5(0-34.75)$ & $12.5(0-30.5)$ & 0.4 \\
\hline & $\mathrm{d}$ & $2.4(0-6.42)$ & $2.85(0-8.78)$ & $1.58(0-5.64)$ & $2.05(0-5.2)$ & 0.5 \\
\hline \multirow[t]{2}{*}{ Evade } & $\mathrm{f}$ & $1(0-4.75)$ & $0(0-1.5)$ & $4(.75-10)$ & $0(0-1.75)$ & $8.3^{*}$ \\
\hline & $\mathrm{d}$ & $1.3(0-10.29)$ & $0(0-1.80)$ & $12.36(0.7-22.59)$ & $0(0-5.87)$ & 7.5 \\
\hline \multirow[t]{2}{*}{ Defensive sideways } & $\mathrm{f}$ & $0.5(0-3.25)$ & $0(0-1.25)$ & $1.5(0-8.75)$ & $0.5(0-1.25)$ & 2.5 \\
\hline & $\mathrm{d}$ & $2.23(0-11.56)$ & $0(0-2.98)$ & $2.88(0-35.35)$ & $0.53(0-4.47)$ & 2.4 \\
\hline \multirow[t]{2}{*}{ Defensive upright } & $\mathrm{f}$ & $0(0-1)$ & $0(0-0)$ & $0(0-3)$ & $0.5(0-2.25)$ & 3.8 \\
\hline & $\mathrm{d}$ & $0(0-2.14)$ & $0(0-0)$ & $0(0-5.36)$ & $0.72(0-7.1)$ & 4 \\
\hline \multirow[t]{2}{*}{ Submissive upright } & $\mathrm{f}$ & $0(0-0)$ & $0(0-0)$ & $0(0-0)$ & $0(0-0)$ & 0 \\
\hline & $\mathrm{d}$ & $0(0-0)$ & $0(0-0)$ & $0(0-0)$ & $0(0-0)$ & 0 \\
\hline \multirow[t]{2}{*}{ Frozen crouch } & $\mathrm{f}$ & $0(0-0.25)$ & $0(0-10.75)$ & $1(0-4.5)$ & $0(0-0.5)$ & 4.6 \\
\hline & $\mathrm{d}$ & $0(0-2.35)$ & $0(0-117.01)$ & $1.07(0-23.61)$ & $0(0-1.43)$ & 3.9 \\
\hline
\end{tabular}

Data expressed as median (lower to upper quartiles) for frequency (f) and duration (d); Significant values refer to Mann-Whitney comparisons with vehicle control; * significance at $\mathrm{p}<0.05$,_significance at $\mathrm{p}<0.02$, \#significance at $\mathrm{p}<0.002$. 
Table 2. Behaviour of untreated intruders as a function of drug state of residents (0.01 - $0.5 \mathrm{mg} / \mathrm{kg} \mathrm{RS} \mathrm{67333).}$

\begin{tabular}{|c|c|c|c|c|c|c|c|}
\hline Behaviours & & Vehicle & $0.01 \mathrm{mg} / \mathrm{kg}$ & $0.1 \mathrm{mg} / \mathrm{kg}$ & $0.5 \mathrm{mg} / \mathrm{kg}$ & $\mathbf{H}$ values & \\
\hline \multirow[t]{2}{*}{ Cage exploration } & $\mathrm{f}$ & $62.5(52.75-75.25)$ & $64(50-75)$ & $57.5(45.75-70.25)$ & $62.5(53.25-72.75)$ & 1.2 & \\
\hline & $\mathrm{d}$ & $186.03(154.55-217.46)$ & $207(174.65-250.23)$ & 199.59 (151.46 - 219.7) & $180.27(132.68-253.55)$ & 1.2 & \\
\hline \multirow[t]{2}{*}{ Rearing } & $\mathrm{f}$ & $37.5(35.75-57.25)$ & $45(20.75-57.5)$ & $38.5(20.25-64)$ & $42.5(23.25-55.5)$ & 0.1 & \\
\hline & $\mathrm{d}$ & $82.37(62.17$ - 127.78) & $82.03(40.22-130.81)$ & $76.14(43.83-138.79)$ & $107.32(46.5-142.92)$ & 0.6 & $p=0.0117$ \\
\hline \multirow[t]{2}{*}{ Maintenance } & $\mathrm{f}$ & $4(2.75-4.5)$ & $2.5(1-4.5)$ & $3(0.75-5.25)$ & $3.5(2-6.5)$ & 2.6 & \\
\hline & d & $19.74(7.85-53.05)$ & $13.25(6.27-16.20)$ & $19.36(1.11-29.97)$ & $16.67(7.69-54.76)$ & 2.4 & \\
\hline \multirow[t]{2}{*}{ Digging } & $\mathrm{f}$ & $22(14-33.5)$ & $11(6-21.75)$ & $20(11.25-24.75)$ & $18.5(6.75-26.5)$ & 2.1 & \\
\hline & d & $60.31(39.82-97.5)$ & $24.24(15.17-42.55)$ & $37.44(21.43-92.33)$ & $51.57(13.97-78.57)$ & 3 & \\
\hline \multirow[t]{2}{*}{ Naso genital } & $\mathrm{f}$ & $4(0.75-9.5)$ & $4.5(1.75-6.5)$ & $5(1.75-7.5)$ & $3.5(1-6.25)$ & 1 & \\
\hline & d & $15.15(1.81-29)$ & $10.55(4.55-25.1)$ & $10.69(3.27-25.71)$ & $8(4.23-20.35)$ & 0.2 & \\
\hline \multirow[t]{2}{*}{ Naso nasal } & $\mathrm{f}$ & $10.5(8.5-13.5)$ & $15.5(13-21.25)^{*}$ & $13(7.75-13.25)$ & $14.5(10.5-21.5)$ & $8.7 *$ & \\
\hline & d & $33.95(18.18-50.52)$ & $56.20(41.33-64.86)$ & $36.71(24.06-43.25)$ & $46.52(29.55-63.7)$ & 6.3 & \\
\hline Non specific & $\mathrm{f}$ & $11(5-19.25)$ & $12(7-18.5)$ & $6.5(5-10.25)$ & $6.5(4.75-9)$ & 6 & \\
\hline investigation & $\mathrm{d}$ & $23.55(15.92-56.80)$ & $47.32(16.38-65.14)$ & $18.13(12.26-26.26)$ & $21.2(14.13-28.81)$ & 5.8 & \\
\hline \multirow[t]{2}{*}{ Follow } & $\mathrm{f}$ & $0.5(0-1.5)$ & $1(0-2.25)$ & $1(0-5)$ & $0(0-1.75)$ & 1.6 & \\
\hline & d & $0.58(0-4.31)$ & $1.51(0-4.1)$ & $3.96(0-7.78)$ & $0(0-3.18)$ & 2.2 & \\
\hline \multirow[t]{2}{*}{ Attend } & $\mathrm{f}$ & $6(2.5-7)$ & $3.5(2.5-10.25)$ & $6(3.75-10)$ & $5.5(1.75-8.5)$ & 0.9 & \\
\hline & d & $6.76(2.34-8.62)$ & $4.89(2.72-11.79)$ & $6.62(3.39-14.32)$ & $7.92(3.25-19.17)$ & 1.6 & \\
\hline \multirow[t]{2}{*}{ Stretched attend } & $\mathrm{f}$ & $2.5(1.75-3.25)$ & $3.5(1.75-6.75)$ & $1.5(0.75-4.5)$ & $2.5(0-6.25)$ & 1.1 & \\
\hline & d & $3.29(2.01-6.21)$ & $4.64(1.79-9.87)$ & $1.92(1.03-7.01)$ & $3.08(0-8.52)$ & 0.8 & \\
\hline \multirow[t]{2}{*}{ Aggressive groom } & $\mathrm{f}$ & $0(0-0)$ & $0(0-0)$ & $0(0-1)$ & $0(0-0)$ & 6.6 & \\
\hline & d & $0(0-0)$ & $0(0-0)$ & $0(0-1.87)$ & $0(0-0)$ & 6.4 & \\
\hline \multirow[t]{2}{*}{ Tail rattle } & $\mathrm{f}$ & $0(0-3.25)$ & $0(0-0)$ & $2.5(0-6.25)$ & $0.5(0-4.5)$ & 6.7 & \\
\hline & d & $0(0-3.94)$ & $0(0-0)$ & $3.38(0-15.64)$ & $1.45(0-9.22)$ & 7.1 & \\
\hline \multirow[t]{2}{*}{ Offensive sideways } & $\mathrm{f}$ & $0.5(0-2)$ & $0(0-1.25)$ & $0(0-3)$ & $0(0-0.25)$ & 2.2 & \\
\hline & d & $0.001(0-0.003)$ & $0(0-0.002)$ & $0(0-0.01)$ & $0(0-0.001)$ & 1.6 & \\
\hline \multirow[t]{2}{*}{ Offensive upright } & $\mathrm{f}$ & $0(0-1)$ & $0(0-0)$ & $3(0-4.25)^{*}$ & $0.5(0-2.25)$ & $12.3_{-}$ & \\
\hline & d & $0(0-2.1)$ & $0(0-0)$ & $8.38(0-20.13)^{*}$ & $0.91(0-6.79)$ & $10.8_{-}$ & \\
\hline \multirow[t]{2}{*}{ Chase } & $\mathrm{f}$ & $0(0-0.75)$ & $0(0-0)$ & $2(0-4.75)$ & $0(0-0.5)$ & 12.6 & \\
\hline & d & $0(0-1.33)$ & $0(0-0)$ & $4.8(0-10.25)$ & $0(0-0.5)$ & 14 & \\
\hline \multirow[t]{2}{*}{ Bite attack } & $\mathrm{f}$ & $0(0-5.25)$ & $0.5(0-5.25)$ & $7(0.75-30.75)$ & $1(0-5.5)$ & 5.2 & \\
\hline & d & $0(0-0.77)$ & $0.80(0-0.88)$ & $1.24(0.13-6.15)$ & $0.24(0-1.62)$ & 4.7 & \\
\hline \multirow[t]{2}{*}{ Evade } & $\mathrm{f}$ & $1.5(0-3.75)$ & $1(0-5.5)$ & $1(0-8.25)$ & $2(0-4.5)$ & 0.3 & \\
\hline & d & $2.14(0-5.31)$ & $0.63(0-7.55)$ & $1.73(0-14.55)$ & $1.75(0-6.1)$ & 0.4 & \\
\hline \multirow[t]{2}{*}{ Defensive upright } & $\mathrm{f}$ & $2(0-19.25)$ & $10(0-24.25)$ & $7.5(0-21.25)$ & $5(1.5-18.25)$ & 0.3 & \\
\hline & $\mathrm{d}$ & $13.97(0-68.76)$ & $31.94(0$ - 93.69) & $32.13(0-78.08)$ & $21.45(3.54-88.71)$ & 0.4 & \\
\hline \multirow[t]{2}{*}{ Defensive sideways } & $\mathrm{f}$ & $3(0-7.25)$ & $3(0-7.75)$ & $2(0-6)$ & $1.5(0-3.25)$ & 0.5 & \\
\hline & d & $6.65(0-23.56)$ & $3.40(0-18.26)$ & $5.69(0-17.53)$ & $3.3(0-7.4)$ & 0.8 & \\
\hline \multirow[t]{2}{*}{ Submissive upright } & $\mathrm{f}$ & $0(0-1.5)$ & $5(0-9)$ & $0.5(0-3.75)$ & $1(0-5.25)$ & 4.1 & \\
\hline & d & $0(0-5.76)$ & $15.26(0-45.11)$ & $2.99(0-18.90)$ & $0.85(0-15.59)$ & 3.4 & \\
\hline \multirow[t]{2}{*}{ Frozen crouch } & $\mathrm{f}$ & $0.5(0-1)$ & $0(0-2.5)$ & $0(0-1.5)$ & $0.5(0-1)$ & 0.6 & \\
\hline & d & $0.41(0-2.28)$ & $0(0-10.71)$ & $0(0-6.65)$ & $0.77(0-2.02)$ & 0.5 & \\
\hline
\end{tabular}

Data expressed as median (lower to upper quartiles) for frequency (f) and duration (d); Significant values refer to Mann-Whitney comparisons with vehicle control; *significance at $\mathrm{p}<0.05$, significance at $\mathrm{p}<0.02$, \#significance at $\mathrm{p}<0.002$. 
The frequency and duration of offensive behaviour were significantly enhanced at $0.1 \mathrm{mg} / \mathrm{kg}(\mathrm{p}<0.02$ [f] \& $\mathrm{p}<$ $00.5[\mathrm{~d}])$.

Further analysis of chase behaviour revealed that variation occurred between drug treated groups although the frequency and duration of evade were enhanced at 0.1 $\mathrm{mg} / \mathrm{kg}$.

While no other significant variation was detected in the offensive category there were non significant enhancements at $0.1 \mathrm{mg} / \mathrm{kg}$.

\subsubsection{RS 67333 and Intruder Defensive Behaviour}

Intruder defensive behaviour remained stable across all doses for both frequency and duration with non signifycant increases and decreases being detected $(\mathrm{H} \leq 4.1, \mathrm{p}>$ $0.05 \& \mathrm{H} \leq 3.4, \mathrm{p}<0.05)$.

\subsection{Resident Behaviours after Treatment with RS 39604 (0.01 - $1 \mathrm{mg} / \mathrm{kg})$ (Table 3)}

\subsubsection{RS 39604 and non Social Behaviour}

Analysis of the non social repertoire revealed no signifycant variation in the frequency or duration of the any element $(\mathrm{H} \leq 3.21, \mathrm{p}>0.05[\mathrm{f}] \& \mathrm{H} \leq 3.84, \mathrm{p}>0.05[\mathrm{~d}])$.

\subsubsection{RS 39604 and Social Behaviour}

Following behaviour showed significant variation for both frequency and duration $(\mathrm{H}=11.26, \mathrm{p}<0.02$ [f] \& H $=11.84, \mathrm{p}<0.02)$. Mann Whitney analysis revealed that the frequency and duration of following were reduced at $1.0 \mathrm{mg} / \mathrm{kg}(\mathrm{p}<0.002)$ in both cases. The frequency and duration of the remainder of the social behaviours in the intruders showed no significant variation across doses $(\mathrm{H}$ $\leq 6.86, \mathrm{p}>0.05$ [f] \& $\mathrm{H} \leq 6.73, \mathrm{p}>0.05$ [d]).

\subsubsection{RS 39604 and Offensive Behaviour}

One way analysis of variance revealed a significant change both the frequency and duration of aggressive grooming $(\mathrm{H}=9, \mathrm{p}<0.05$ [f] \& $\mathrm{H}=8.4, \mathrm{p}<0.05$ [d]). The frequency of aggressive grooming was decreased at 0.01 $\mathrm{mg} / \mathrm{kg}(\mathrm{p}<0.02)$ while the duration was decreased at 0.1 $\mathrm{mg} / \mathrm{kg}(\mathrm{p}<0.02)$. No other elements were significantly modified in this category $(\mathrm{H} \leq 4.57, \mathrm{P}>0.05[\mathrm{f}] \& \mathrm{H} \leq$ 4.67, $\mathrm{p}<0.05[\mathrm{~d}])$.

\subsubsection{RS 39604 and Defensive Behaviour}

A significant change in the frequency of frozen crouch behaviour $(\mathrm{H}=9, \mathrm{p}<0.05[\mathrm{f}] \& \mathrm{H}=8.4, \mathrm{p}<0.05$ [d]). This behaviour was found to be enhanced at $0.01 \mathrm{mg} / \mathrm{kg}$ $(\mathrm{p}<0.02)$. No other elements of defensive behaviour were significantly $(\mathrm{H} \leq 6.15, \mathrm{p}>0.05[\mathrm{f}] \&[\mathrm{~d}])$.

\subsection{Intruder Behaviours as a Function of Resident Behaviour after Treatment with RS 39604 (0.01 mg/kg - 1 mg/kg) (Table 4)}

\subsubsection{RS 39604 and Non-Social Behaviour}

RS 39604 produced no significant changes in the fre- quency or duration of non social behaviour in the intruder group $(\mathrm{H} \leq 4.79, \mathrm{p}>0.05[\mathrm{f}] \& \mathrm{H} \leq 4.87, \mathrm{p}>0.05$ [d]).

\subsubsection{RS 39604 and Social Behaviour}

Analysis revealed significant variation in the frequency of naso naso sniffing $(\mathrm{H}=7.92, \mathrm{p}<0.05)$ and naso genital sniffing $(\mathrm{H}=8.59, \mathrm{p}<0.05)$. Significant variation was detected in the duration of nsi $(\mathrm{H}=10.17, \mathrm{p}<0.02)$ while both the frequency and duration of following were also significantly modified $(\mathrm{H}=10.13, \mathrm{p}<0.02[\mathrm{f}] \& \mathrm{H}$ $=8.54, \mathrm{p}<0.05[\mathrm{~d}])$.

The frequency of naso genital sniffing was increased at $0.01 \mathrm{mg} / \mathrm{kg}(\mathrm{p}<0.02)$ and $0.1 \mathrm{mg} / \mathrm{kg}(\mathrm{p}<0.02)$. The frequency of naso naso sniffing and the duration of nsi were also increased at $0.1 \mathrm{mg} / \mathrm{kg}(\mathrm{p}<0.02$ in both cases). The frequency and duration of following also showed a significant enhancement at $0.1 \mathrm{mg} / \mathrm{kg}(\mathrm{p}<0.05$ in both cases).

No other aspects of social behaviour were modified $(\mathrm{H}$ $\leq 4.12, \mathrm{p}>0.05[\mathrm{f}] \& \mathrm{H} \leq 4.83, \mathrm{p}>0.05[\mathrm{~d}])$.

\subsubsection{RS 39604 and Offensive Behaviour}

Kruskal Wallis analysis revealed significant variation in the frequency and duration of aggressive grooming $(\mathrm{H}=$ $8.27, \mathrm{p}<0.05$ [f] \& $\mathrm{H}=8.1, \mathrm{p}<0.05$ [d]). Further analysis showed an increase in this behaviour at $0.1 \mathrm{mg} / \mathrm{kg}(\mathrm{p}$ $<0.02$ [f] \& [d]) and at $1 \mathrm{mg} / \mathrm{kg}(\mathrm{p}<0.05$ [f] \& [d]).

While significant variation was detected for offensive sideways behaviour variation was found to occur between treatment groups and not with controls.

\subsubsection{RS 39604 and Defensive Behaviour}

Intruder defensive behaviour showed no significant variation follow resident treatment with RS $39604(\mathrm{H} \leq 6.15$, $\mathrm{p}>0.05[\mathrm{f}] \&[\mathrm{~d}])$.

\section{Discussion}

RS 67333 failed to produce any significant modification of resident behaviour in this model of aggression. Non social behaviour was generally reduced by administration of RS 67333 but to a non significant degree. Thus it must be proposed that RS 67333 possesses no sedative effects in this model.

RS 67333 did not largely affect social behaviour in this model. Only one aspect of social behaviour was significantly modified by the compound. The frequency and duration of stretched attend were significantly enhanced at $0.1 \mathrm{mg} / \mathrm{kg}$.

The only other element of the behavioural repertoire that was significantly changed was the frequency of evade behaviour. While this variation occurred within the drug treated groups and not with controls, the frequency and duration of evade behaviour were enhanced at 0.1 
Table 3. Effects of RS $39604(0.01-1.0 \mathrm{mg} / \mathrm{kg})$ on behaviours displayed by resident mice.

\begin{tabular}{|c|c|c|c|c|c|c|c|}
\hline Behaviors & & Vehicle & $0.01 \mathrm{mg} / \mathrm{kg}$ & $0.1 \mathrm{mg} / \mathrm{kg}$ & $1.0 \mathrm{mg} / \mathrm{kg}$ & $\mathbf{H}$ values & \\
\hline \multirow[t]{2}{*}{ Cage exploration } & $\mathrm{f}$ & $60.5(47-95.75)$ & $67(53-80.75)$ & $67(58-81.75)$ & $65.5(48.25-83.25)$ & 0.29 & \\
\hline & $\mathrm{d}$ & $220.65(141.75-275.42)$ & $321.98(174.92-340.91)$ & $262.06(202.59-308.81)$ & $290.24(170.72-334.82)$ & 3.84 & \\
\hline \multirow[t]{2}{*}{ Rearing } & $\mathrm{f}$ & $41(27.75-67.25)$ & $31.5(15-49.25)$ & $33.5(26.5-48)$ & $29(20.75-42.5)$ & 3.21 & \\
\hline & d & $70.4(40.7-116.17)$ & $49.77(20.46-82.96)$ & $52.98(40.07-67.14)$ & $50.31(34.22-65.93)$ & 2.52 & \\
\hline \multirow[t]{2}{*}{ Maintenance } & $\mathrm{f}$ & $3(1-4)$ & $2(1.75-4.5)$ & $2.5(1-3.25)$ & $2(0-7.25)$ & 0.68 & \\
\hline & d & $7.77(3.77-10.24)$ & $7.79(5.41-17.93)$ & $10.79(3.23-17)$ & $5.38(0-27.7)$ & 1.19 & \\
\hline \multirow[t]{2}{*}{ Digging } & $\mathrm{f}$ & $0.5(0-3.25)$ & $0.5(0-1.25)$ & $0(0-1)$ & $0(0-0.5)$ & 2.25 & \\
\hline & d & $0.69(0-7.97)$ & $0.47(0-2.32)$ & $0(0-1)$ & $0(0-0.94)$ & 2.96 & \\
\hline \multirow[t]{2}{*}{ Naso-genital } & $\mathrm{f}$ & $16.5(9.75-20.75)$ & $20(11.75-25)$ & $16(8.75-27)$ & $8.5(5.75-12.75)$ & 6.86 & \\
\hline & d & $46.65(17.2-66.74)$ & $55.68(28.14-91.12)$ & $59.12(26.85-76.95)$ & $20.39(15.02-28.32)$ & 6.73 & \\
\hline \multirow[t]{2}{*}{ Naso-nasal } & $\mathrm{f}$ & $15.5(11.5-19.25)$ & $16(13-21)$ & $19(17-22.5)^{*}$ & $16(12.75-23)$ & 4.47 & \\
\hline & d & $45.48(25.56-54.9)$ & $36.66(33.16-61.74)$ & $55.18(44.49-66.05)$ & $48.1(28.77-64.04)$ & 3.21 & \\
\hline Non-specific & f & $35(15.5-42.25)$ & $26(22-35)$ & $25.5(19.75-29)$ & $24.5(15.25-28.25)$ & 2.43 & \\
\hline investigation & d & $101.79(49.24-142.02)$ & $65.37(53.56-94.97)$ & $74.96(52.8-103.15)$ & $63.77(35.71-84.73)$ & 2.38 & \\
\hline \multirow[t]{2}{*}{ Follow } & $\mathrm{f}$ & $7.5(2.75-11.25)$ & $4.5(2.75-6.5)$ & $4.5(2.75-10)$ & $1.5(0-3) \dagger$ & 11.26 & $\mathrm{p}=0.0104$ \\
\hline & d & $10.24(3.03-16.47)$ & $7.01(3.73-12.21)$ & $6.96(3.92-17.99)$ & $2.42(0-3.87) \dagger$ & 11.84 & $\mathrm{p}=0.0079$ \\
\hline \multirow[t]{2}{*}{ Attend/approach } & $\mathrm{f}$ & $8.5(4.25-11)$ & $3(2-7)$ & $6.5(2.75-9.5)$ & $5(2.75-8.25)$ & 3.24 & \\
\hline & d & $9.3(5.84-12.65)$ & $4.21(2.41-6.08)$ & $8.52(5.4-12.05)$ & $5.07(2.31-13.77)$ & 4.12 & \\
\hline \multirow[t]{2}{*}{ Stretch/attend } & $\mathrm{f}$ & $1.5(0.75-2.5)$ & $1(0-3.5)$ & $0.5(0-2.25)$ & $1.5(0-3.25)$ & 1.06 & \\
\hline & d & $1.87(0.82-2.9)$ & $1.21(0-3.5)$ & $0.97(0-2.65)$ & $1.42(0-4)$ & 0.7 & \\
\hline \multirow[t]{2}{*}{ Aggressive groom } & f & $0.5(0-2)$ & $0(0-0)^{*}$ & $0(0-0.25)$ & $0(0-0) \dagger$ & 9 & $\mathrm{p}=0.0293$ \\
\hline & d & $0.55(0-7.24)$ & $0(0-0)$ & $0(0-1.04)$ & $0(0-0) \dagger$ & 8.4 & $\mathrm{p}=0.0384$ \\
\hline \multirow[t]{2}{*}{ Tail rattle } & $\mathrm{f}$ & $2(0-6)$ & $0(0-0)$ & $0(0-1.75)$ & $0.5(0-20)$ & 4.57 & \\
\hline & d & $2.09(0-8.61)$ & $0(0-0)$ & $0(0-1.7)$ & $0.29(0-23.28)$ & 4.67 & \\
\hline \multirow[t]{2}{*}{ Offensive sideways } & f & $0(0-6.25)$ & $0(0-1.75)$ & $0(0-7)$ & $0(0-27.25)$ & 0.87 & \\
\hline & d & $0(0-18.73)$ & $0(0-3.05)$ & $0(0-21.23)$ & $0(0-55.09)$ & 0.72 & \\
\hline \multirow[t]{2}{*}{ Offensive upright } & $\mathrm{f}$ & $0(0-4.5)$ & $0(0-0.5)$ & $0(0-1.5)$ & $0(0-5)$ & 0.99 & \\
\hline & d & $0(0-16.11)$ & $0(0-0.52)$ & $0(0-3.03)$ & $0(0-8.43)$ & 1 & \\
\hline \multirow[t]{2}{*}{ Chase } & $\mathrm{f}$ & $0(0-0.25)$ & $0(0-0)$ & $0(0-1)$ & $0(0-0.25)$ & 1.86 & \\
\hline & d & $0(0-0.1)$ & $0(0-0)$ & $0(0-1.27)$ & $0(0-0.48)$ & 2.45 & \\
\hline \multirow[t]{2}{*}{ Bite attack } & $\mathrm{f}$ & $0(0-8)$ & $0(0-4)$ & $0(0-5.75)$ & $0(0-33)$ & 1.18 & \\
\hline & d & $0(0-1.67)$ & $0(0-1.67)$ & $0(0-1.53)$ & $0(0-6.11)$ & 0.87 & \\
\hline \multirow[t]{2}{*}{ Evade } & $\mathrm{f}$ & $0(0-0.25)$ & $0(0-0.25)$ & $0(0-0)$ & $0(0-2.75)$ & 3.38 & \\
\hline & d & $0(0-0.56)$ & $0(0-0.81)$ & $0(0-0)$ & $0(0-4.8)$ & 3.25 & \\
\hline \multirow[t]{2}{*}{ Defensive sideways } & $\mathrm{f}$ & $0(0-0.25)$ & $0(0-0.25)$ & $0(0-0)$ & $0(0-1.25)$ & 3.14 & \\
\hline & d & $0(0-0.29)$ & $0(0-0.43)$ & $0(0-0)$ & $0(0-1.53)$ & 3.05 & \\
\hline \multirow[t]{2}{*}{ Defensive upright } & $\mathrm{f}$ & $0(0-0)$ & $0(0-0)$ & $0(0-0)$ & $0(0-0.5)$ & 6.15 & \\
\hline & d & $0(0-0)$ & $0(0-0)$ & $0(0-0)$ & $0(0-0.88)$ & 6.15 & \\
\hline \multirow[t]{2}{*}{ Submissive upright } & $\mathrm{f}$ & $0(0-0)$ & $0(0-0)$ & $0(0-0)$ & $0(0-0)$ & 0 & \\
\hline & d & $0(0-0)$ & $0(0-0)$ & $0(0-0)$ & $0(0-0)$ & 0 & \\
\hline \multirow[t]{2}{*}{ Frozen crouch } & $\mathrm{f}$ & $0(0-0)$ & $0(0-2.5)$ & $2(0-3.25) \dagger$ & $0(0-1.25)$ & 7.88 & $\mathrm{p}=0.0483$ \\
\hline & $\mathrm{d}$ & $0(0-0.14)$ & $0(0-16.82)$ & $11.42(0-29.08)$ & $0(0-3.17)$ & 7.02 & \\
\hline
\end{tabular}

Data expressed as medians (lower to upper quartiles) for frequency (f) and duration (d); Significant values refer to Mann-Whitney comparisons with vehicle. *p $<0.05, \uparrow \mathrm{p}<0.02, \# \mathrm{p}<0.002$. 
Table 4. Behaviour of untreated intruders as a function of drug state of residents (0.01 - $1.0 \mathrm{mg} / \mathrm{kg} \mathrm{RS} \mathrm{39604).}$

\begin{tabular}{|c|c|c|c|c|c|c|c|}
\hline Behaviors & & Vehicle & $0.01 \mathrm{mg} / \mathrm{kg}$ & $0.1 \mathrm{mg} / \mathrm{kg}$ & $1.0 \mathrm{mg} / \mathrm{kg}$ & $H$ values & \\
\hline \multirow[t]{2}{*}{ Cage exploration } & $\mathrm{f}$ & $93.5(78.75-104.75)$ & $95(82.25-110)$ & $88.5(84-93.25)$ & $77(48.25-95)$ & 4.79 & \\
\hline & $\mathrm{d}$ & $247.1(212.34-304.46)$ & $285.05(219.83-289.36)$ & $261.2(224.74-290.66)$ & $204.28(147.66-268.47)$ & 4.87 & \\
\hline \multirow[t]{2}{*}{ Rearing } & $\mathrm{f}$ & $73(60.5-90.5)$ & $63(56-76.25)$ & $66(56.75-76.5)$ & $50(27.5-68.25)$ & 4.28 & \\
\hline & $\mathrm{d}$ & $117.6(88.17-158.83)$ & $110.12(100.97-119.36)$ & $120.48(73.58-154.47)$ & $93.23(37.34-126.29)$ & 2.41 & \\
\hline \multirow[t]{2}{*}{ Maintenance } & $\mathrm{f}$ & $6(3-9.75)$ & $5(3-8.5)$ & $4.5(3-7)$ & $5(2.5-8.25)$ & 0.55 & \\
\hline & $\mathrm{d}$ & $15.96(12.64-91.2)$ & $17.3(11.1-34)$ & $21.9(17.1-25.58)$ & $17.44(12.68-41.7)$ & 0.54 & \\
\hline \multirow[t]{2}{*}{ Digging } & $\mathrm{f}$ & $22.5(10.25-38)$ & $20(17.25-30.75)$ & $20(14.25-24)$ & $13.5(2.5-24.75)$ & 2.61 & \\
\hline & $\mathrm{d}$ & $71.21(24.44-116.89)$ & $66.96(40.01-122.97)$ & $55.62(44.47-87.28)$ & $30.12(5.83-108.84)$ & 1.15 & \\
\hline \multirow[t]{2}{*}{ Naso-genital } & $\mathrm{f}$ & $2.5(1-3.25)$ & $5(3.75-8) \dagger$ & $7(2.75-12.25) \dagger$ & $2(1-8)$ & 7.92 & $\mathrm{p}=0.0478$ \\
\hline & $\mathrm{d}$ & $4.45(3.24-8.55)$ & $12.03(8.31-24.38)^{*}$ & $18.74(6.44-31.63)^{*}$ & $7.41(0.96-17.79)$ & 7.02 & \\
\hline \multirow[t]{2}{*}{ Naso-nasal } & $\mathrm{f}$ & $7.5(4.75-11)$ & $12(5.75-16.5)$ & $17(12-19.75) \dagger$ & $16(7.5-17)$ & 8.59 & $\mathrm{p}=0.0353$ \\
\hline & $\mathrm{d}$ & $15.02(11.13-39.52)$ & $25.81(19.33-34.48)$ & $34.49(25.41-55.01)$ & $34.1(22.32-54.6)$ & 4.83 & \\
\hline Non-specific & $\mathrm{f}$ & $5.5(2.75-11)$ & $12.5(6.5-15.25)$ & $13(10.5-16)^{*}$ & $6(5-13.75)$ & 6.82 & \\
\hline investigation & d & $13.94(4.6-20.22)$ & $25.25(16.25-33.19)$ & $30.76(24.22-46.41) \dagger$ & $18.93(14.78-35.7)$ & 10.17 & $\mathrm{p}=0.0172$ \\
\hline \multirow[t]{2}{*}{ Follow } & $\mathrm{f}$ & $0(0-0.25)$ & $1(0-1.5)$ & $2(0-2.25)^{*}$ & $0(0-0.25)$ & 10.13 & $\mathrm{p}=0.0175$ \\
\hline & $\mathrm{d}$ & $0(0-0.81)$ & $0.74(0-3.14)$ & $2.51(0-3.52)^{*}$ & $0(0-0.39)$ & 8.54 & $\mathrm{p}=0.036$ \\
\hline \multirow[t]{2}{*}{ Attend/approach } & $\mathrm{f}$ & $0.5(0-1)$ & $1.5(0-4.25)$ & $3(0-4)$ & $2(0.75-5)$ & 4.12 & \\
\hline & $\mathrm{d}$ & $0.42(0-2.38)$ & $1.32(0-4.67)$ & $2.45(0-3.64)$ & $3.19(0.41-5.95)$ & 2.36 & \\
\hline \multirow[t]{2}{*}{ Stretch/attend } & $\mathrm{f}$ & $0(0-0)$ & $0(0-0.25)$ & $0(0-0)$ & $0(0-2)^{*}$ & 5.92 & \\
\hline & $\mathrm{d}$ & $0(0-0)$ & $0(0-0.17)$ & $0(0-0)$ & $0(0-2.16)^{*}$ & 6.01 & \\
\hline \multirow[t]{2}{*}{ Aggressive groom } & $\mathrm{f}$ & $0(0-0)$ & $0(0-1)$ & $1(0-2) \dagger$ & $0(0-1)^{*}$ & 8.27 & $\mathrm{p}=0.0408$ \\
\hline & $d$ & $0(0-0)$ & $0(0-2.73)$ & $2.01(0-7.02) \dagger$ & $0(0-3.8)^{*}$ & 8.1 & $\mathrm{p}=0.044$ \\
\hline \multirow[t]{2}{*}{ Tail rattle } & $\mathrm{f}$ & $0(0-0.25)$ & $0(0-0)$ & $0(0-1)$ & $0(0-2.75)$ & 4.99 & \\
\hline & $\mathrm{d}$ & $0(0-0.21)$ & $0(0-0)$ & $0(0-0.47)$ & $0(0-3.15)$ & 4.95 & \\
\hline \multirow[t]{2}{*}{ Offensive sideways } & $\mathrm{f}$ & $0(0-0)$ & $0(0-0)$ & $0(0-0)$ & $0.5(2.25)$ & 9.21 & $\mathrm{p}=0.0267$ \\
\hline & d & $0(0-0.2)$ & $0(0-0)$ & $0(0-0)$ & $0.38(0-2.65)$ & 8.1 & $\mathrm{p}=0.044$ \\
\hline \multirow[t]{2}{*}{ Offensive upright } & $\mathrm{f}$ & $0(0-0.25)$ & $0(0-0)$ & $0(0-0)$ & $0(0-4.75)$ & 5.98 & \\
\hline & $\mathrm{d}$ & $0(0-0.96)$ & $0(0-0)$ & $0(0-0)$ & $0(0-8.52)$ & 5.98 & \\
\hline \multirow[t]{2}{*}{ Chase } & $\mathrm{f}$ & $0(0-0)$ & $0(0-0.25)$ & $0(0-0)$ & $0(0-3)$ & 6.03 & \\
\hline & $\mathrm{d}$ & $0(0-0)$ & $0(0-0.32)$ & $0(0-0)$ & $0(0-2.25)$ & 6.08 & \\
\hline \multirow[t]{2}{*}{ Bite attack } & $\mathrm{f}$ & $0(0-1.25)$ & $0(0-1.25)$ & $0(0-1)$ & $1(0-8.75)$ & 3.29 & \\
\hline & $\mathrm{d}$ & $0(0-0.29)$ & $0(0-0.74)$ & $0(0-0.25)$ & $0.2(0-1.2)$ & 2.72 & \\
\hline \multirow[t]{2}{*}{ Evade } & $\mathrm{f}$ & $0(0-2.25)$ & $0(0-0.25)$ & $0(0-0.5)$ & $0(0-5.25)$ & 1.46 & \\
\hline & d & $0(0-1.44)$ & $0(0-0.1)$ & $0(0-0.58)$ & $0(0-5.87)$ & 1.99 & \\
\hline \multirow[t]{2}{*}{ Defensive sideways } & $\mathrm{f}$ & $0(0-2)$ & $0(0-1.75)$ & $0(0-2.25)$ & $0(0-6.25)$ & 0.79 & \\
\hline & $\mathrm{d}$ & $0(0-3.09)$ & $0(0-6.77)$ & $0(0-6.24)$ & $0(0-12.89)$ & 0.6 & \\
\hline \multirow[t]{2}{*}{ Defensive upright } & $\mathrm{f}$ & $0(0-8.5)$ & $0(0-0.75)$ & $0(0-5.25)$ & $0(0-26.25)$ & 1.77 & \\
\hline & $\mathrm{d}$ & $0(0-34.17)$ & $0(0-1.69)$ & $0(0-15.42)$ & $0(0-100.67)$ & 1.87 & \\
\hline \multirow[t]{2}{*}{ Submissive upright } & $\mathrm{f}$ & $0(0-1)$ & $0(0-0)$ & $0(0-0)$ & $0(0-0.25)$ & 6.15 & \\
\hline & $\mathrm{d}$ & $0(0-0)$ & $0(0-0)$ & $0(0-0)$ & $0(0-1.43)$ & 6.15 & \\
\hline \multirow[t]{2}{*}{ Frozen crouch } & $\mathrm{f}$ & $0(0-1)$ & $0(0-0.25)$ & $0(0-1)$ & $0(0-2)$ & 1.52 & \\
\hline & $\mathrm{d}$ & $0(0-1.78)$ & $0(0-0.92)$ & $0(0-2.33)$ & $0(0-9.87)$ & 0.99 & \\
\hline
\end{tabular}

Data expressed as medians (lower to upper quartiles) for frequency (f) and duration (d); Significant values refer to Mann-Whitney comparisons with vehicle. * $<0.05, \uparrow \mathrm{P}<0.02$, \#p $<0.002$. 
$\mathrm{mg} / \mathrm{kg}$. The frequency and duration of defensive behaveiour did show non significant increases at the $0.1 \mathrm{mg} / \mathrm{kg}$ dose. No aspect of offensive behaviour showed any significant change.

While intruder non social and defensive behaviour showed no significant modifications intruder social and offensive behaviour did show significant change in response to the resident animals. The frequency of naso naso sniffing was significantly enhanced at $0.01 \mathrm{mg} / \mathrm{kg}$. All other social behaviours also showed slight enhancements at this same dose level.

Intruder offensive upright attacks showed a significant increase in both frequency and duration at $0.1 \mathrm{mg} / \mathrm{kg}$. Chase behaviour showed significant variation but this occurred within the drug treated groups and not with controls. Both the frequency and duration of chase were enhanced at $0.1 \mathrm{mg} / \mathrm{kg}$.

RS 39604 produced some small changes in behaviour in this model of aggression. No sedative effects were detected for this drug indicated by the lack of effect on non social behaviour. Analysis detected significant changes in social behaviour with enhancements in the frequency and duration of following being detected at $1.0 \mathrm{mg} / \mathrm{kg}$. This was the only aspect of social behaviour that was significantly changed by RS 39604 .

With regard to aggressive behaviour a significant change in the frequency and duration of aggressive grooming was detected at $1 \mathrm{mg} / \mathrm{kg}$. The frequency of this behaviour was also reduced at $0.01 \mathrm{mg} / \mathrm{kg}$. Failure to detect any changes in the other elements of aggressive behaveiour may be a result of low baseline levels of aggression.

There were few changes detected in defensive behaveiour and this set of behaviours also produced low baseline levels of aggression due to the tendency of resident animals to display more attack like behaviours. Only a significant increase in frozen crouch behaviour was detected at $0.1 \mathrm{mg} / \mathrm{kg}$.

Analysis of intruder behaviour produced a greater array of significant results. Although non social behaviour showed no significant modification the frequency of naso naso sniffing, naso genital sniffing and following were all enhanced significantly at $0.1 \mathrm{mg} / \mathrm{kg}$. The duration of non specific investigation and following were also enhanced by RS 39604 at the same dose. These behaviours may be linked to the increase in resident frozen crouch behaviours at the same dose. There was also a low level of resident aggressive behaviour at this dose which would serve to reduce defensive behaviour in the intruder animals. Intruder defensive behaviour was reduced, although non-significantly, at this level.

While there has been no work published regarding the role of $5-\mathrm{HT}_{4}$ receptors in aggressive behaviour, investigations examining the role of $5-\mathrm{HT}_{4}$ receptors in anxiety have reported an increase in social interaction following treatment with 5- $\mathrm{HT}_{4}$ antagonists [10]. Specifically, the $5-\mathrm{HT}_{4}$ antagonist SB 204070A increased rat social behaviour at doses between $0.001 \mathrm{mg} / \mathrm{kg}$ and $0.1 \mathrm{mg} / \mathrm{kg}$; a result indicative of a mild anxiolytic effect. These results supported earlier findings that $5-\mathrm{HT}_{4}$ antagonists produced anxiolytic like effects in rats tested in the elevated plus maze [8]. Combined results from resident and intruder social behaviour in this study also indicated an enhancement of social behaviours at $0.1 \mathrm{mg} / \mathrm{kg}$. While enhancements were largely detected in the intruder animals these behaviours occur in response to changes in behaveioural cues from the residents. These augmentations of social behaviour may support the evidence for mild anxiolytic effects induced by $5-\mathrm{HT}_{4}$ antagonists.

Results from RS 67333 also serve to support these previous findings. The combined increase of stretched attend behaviour (thought to be associated with risk assessment) and the rise in defensive behaviours are suggestive of a weak enhancement of anxiety like behaviours. These changes in resident behaviour also resulted in increased aggressive behaviour from the intruder animals. As RS 67333 was a partial agonist these effects may be enhanced by administration of a full agonist for this site.

With regard to aggressive behaviours RS 39604 produced no significant changes in resident aggressive behaviour. There was an increase in intruder aggressive grooming and a consequent increase in resident frozen crouch behaviour. This may represent a function of increased contact due to increased social behaviour and therefore an increased likelihood of aggressive behaviour. RS 67333 also produced little change in resident aggressive behaviour.

In conclusion, the lack of effects of the $5-\mathrm{HT}_{4}$ receptor agonist and antagonist employed in this study indicates that $5-\mathrm{HT}_{4}$ receptors have no specific role to play in the modulation of murine aggression.

\section{REFERENCES}

[1] R. Bell and H. Hobson, "5-HT $\mathrm{H}_{1 \mathrm{~A}}$ Receptor Influences on Rodent Social and Agonistic Behaviour: A Review and Empirical Study," Neuroscience and Biobehavioural Reviews, Vol. 18, No. 3, 1994, pp. 325-333.

[2] R. Bell, P. Mitchell and H. Hobson, "Effects of the 5-HT $1 \mathrm{~A}$ Antagonist (+)-WAY-100135 on Murine Social and Agonistic Behaviour," Pharmacology, Biochemistry and Behavior, Vol. 54, No. 1, 1996, pp. 159-167. doi:10.1016/0091-3057(95)02168-X

[3] R. Bell, P. Mitchell and K. Lynch, "Lack of Effect of the $5-\mathrm{HT}_{1 \mathrm{~A}}$ Antagonist WAY-100635 on Murine Agonistic Behaviour," Pharmacology, Biochemistry and Behavior, Vol. 64, No. 3, 1999, pp. 549-555. doi:10.1016/S0091-3057(99)00105-7

[4] R. Bell, C. Donaldson and D. Gracey, "Differential Effects of CGS12066B and CP94-253 on Murine Social and 
Agonistic Behaviour," Pharmacology, Biochemistry and Behavior, Vol. 52, No. 1, 1995, pp. 7-16. doi:10.1016/0091-3057(95)00077-A

[5] D. Fontana, S. E. Daniels, E. Wong, R. D. Clark and R. Eglen, "The Effects of Novel Selective 5-Hydroxytryptamine $\left(5-\mathrm{HT}_{4}\right)$ Receptor Ligands in Rat Spatial Navigation," Neuropharmacology, Vol. 36, No. 4-5, 1997, pp. 689-696. doi:10.1016/S0028-3908(97)00055-5

[6] G. Lucas, V. Rymar, J. Du, O. Mnie-Filali, C. Bisgaard, S. Manta, L. Lambas-Senas, O. Wiborg, N. Haddjeri, G. Pineyro, A. F. Sadikot and G. Debonnel, "Serotonin 4 (5-HT 4$)$ Receptor Agonists Are Putative Antidepressants with a Rapid Onset of Action," Neuron, Vol. 55, No. 5, 2007, pp. 712-725. doi:10.1016/j.neuron.2007.07.041

[7] G. Lucas, J. Du, T. Romeas, O. Mnie-Filali, Haddjeri, G. Pineyro and G. Debonnel, "Selective Serotonin Reuptake Inhibitors Potentiate the Rapid Antidepressant-Like Effects of Serotonin 4 Receptor Agonists in the Rat," PLoS
One, Vol. 5, No. 2, 2010, p. e9253. doi:10.1371/journal.pone.0009253

[8] J. S. Silvestre, A. G. Fernandez and J. M. Palacois, "Effects of 5-HT $\mathrm{HT}_{4}$ Receptor Antagonists on Rat Behaviour in the Elevated Plus-Maze Test," European Journal of Pharmacology, Vol. 309, No. 3, 1996, pp. 219-222. doi:10.1371/journal.pone.0009253

[9] H. M. Francis, N. J. Kraushaar, L. R. Hunt and J. L. Cornish, "Serotonin 5-HT 4 Receptors in the Nucleus Accumbens Are Specifically Involved in the Appetite Suppressant and Not Locomotor Stimulant Effects of MDMA ('Ecstasy')," Psychopharmacology, Vol. 213, No. 2-3, 2011, pp. 355-363. doi:10.1007/s00213-010-1982-9

[10] G. A. Kennett, F. Bright, B. Trail, T. P. Blackburn and G. Sanger, "Anxiolytic-Like Actions of the Selective 5-HT Receptor Antagonists SB204070 and SB207266A in Rats," Neuropharmacology, Vol. 36, No. 4-5, 1997, pp. 707-712. doi:10.1016/S0028-3908(97)00037-3 